\title{
Numerical Approximation of a Transport Equation with a Time-Dependent Dispersion Flux
}

\author{
Cidália Neves*, Adérito Araújo ${ }^{\dagger}$ and Ercília Sousa ${ }^{\dagger}$ \\ *ISCAC, Instituto Politécnico de Coimbra, 3040-316 Coimbra, Portugal and CMUC, Department of \\ Mathematics, University of Coimbra, 3001-454 Coimbra, Portugal \\ ${ }^{\dagger}$ CMUC, Department of Mathematics, University of Coimbra, 3001-454 Coimbra, Portugal
}

\begin{abstract}
The objective of this work is to discuss a more general one-dimensional diffusion equation that accounts for certain aspects such as the variation of a parameter that describes the relaxation time of the mass flux and also the presence of a potential field. The equation will have properties similar to a an hyperbolic equation or parabolic equation according to which values of the relaxation parameter or the potential field we consider. In the hyperbolic case we deal with some discontinuities. We apply a numerical scheme to solve this equation, which consists of using an inverse Laplace transform algorithm. The Laplace method is used to remove the time-dependent terms in the governing equation and boundary conditions. For a constant potential field general solutions can be determined. On the other hand for a non-constant potential field, a spatial discretisation must be considered. We will study the convergence of the numerical scheme based on the inverse of Laplace transform and present some test problems.
\end{abstract}

Keywords: Diffusion equation, Inverse Laplace transform, Error analysis PACS: 02.60.Jh, 02.60.Lj (AMS classification 35Q80, 65M12, 65D30)

\section{INTRODUCTION}

Diffusion problems that describe physical phenomenon is widespread in various branches of science and engineering [1]. Most of these problems are described with Fourier's law or Fick's law. The dynamics of a Brownian particle, for instance, is described by

$$
\frac{\partial C}{\partial t}=-D \frac{\partial^{2} C}{\partial x^{2}}
$$

where $C$ is the mass concentration of the Brownian particle and $D$ is the diffusion coefficient. This equation can be derived, at a macroscopic level, by combining the Fick's law of diffusion

$$
J=-D \frac{\partial C}{\partial x}
$$

with the continuity equation

$$
\frac{\partial C}{\partial t}+\frac{\partial J}{\partial x}=0
$$

where $J$ represents the mass flux. When an uniform force field such as gravitation, exists, an uniform flow is produced with the terminal velocity determined by the balance of the driving force and the frictional force from the surrounding fluid acting on the particle [2]. Therefore, the flow is given by

$$
J=-D \frac{\partial C}{\partial x}-P C
$$

where

$$
P=\frac{1}{m \gamma} \frac{d V}{d x},
$$

$V$ is the potential field, $m$ is the particle mass and $\gamma$ the friction constant. The diffusion constant can also be written as given by

$$
D=\kappa_{B} \frac{T}{m \gamma},
$$

CP1048, Numerical Analysis and Applied Mathematics, International Conference 2008

edited by T. E. Simos, G. Psihoyios, and Ch. Tsitouras

(C) 2008 American Institute of Physics 978-0-7354-0576-9/08/\$23.00 
where $\kappa_{B}$ is the Boltzmann constant and $T$ is the temperature of the fluid.

It is well known that these problems may break down, specially if we are interested in the transient problem in an extremely short period of time, or for low temperature [3]. In order to describe the mass diffusion with a finite speed of propagation, we must consider the so-called non-Fickian diffusion equations. Das [4], [5] has derived a nonFickian diffusion equation in the presence of a potential field from the Kramers equation. This equation is a hyperbolic equation although, the corresponding Fickian equation is a parabolic equation.

To accommodate the finite propagation speed, the generalized Fick's law can be written as [5]

$$
\theta \frac{\partial J}{\partial t}+J=-D \frac{\partial C}{\partial x}-P C
$$

where $\theta \in[0,1]$ is the parameter that measures the propagation speed of the mass wave and can be regarded as the relaxation time of the mass flux.

Strack [6] has employed a flux similar to (7) with $P=0$ but in three-dimensional form in order to model the nonFickian behaviour of transport in porous media with small-scale heterogeneities.

Elimination of the mass flux $J$ between equations (3) and (7) leads to the following equation

$$
\frac{\partial C}{\partial t}+\theta \frac{\partial^{2} C}{\partial t^{2}}=\frac{\partial}{\partial x}(P C)+D \frac{\partial^{2} C}{\partial x^{2}}, x \in(0, \infty), t>0 .
$$

For our problem we consider the initial conditions given by

$$
\begin{aligned}
C(x, 0) & =0, \quad x \in[0, \infty) \\
\theta \frac{\partial C(x, 0)}{\partial t} & =0, \quad x \in[0, \infty)
\end{aligned}
$$

and the boundary conditions

$$
\begin{aligned}
C(0, t) & =g(t), \quad t>0 \\
C(\infty, t) & =0, \quad t>0 .
\end{aligned}
$$

Note that equation (8) turns to the classical advection diffusion equation for $\theta=0$ and it is a parabolic equation.

In this work we study equation (8) which is a more general equation than the ones presented in similar works, such as [3] and [7]. This equation takes in consideration two types of fluxes which are caracterised by the parameter $\theta$. The parameter $\theta$ is directly related with the parabolic or hyperbolic behaviour of equation (8). We use a numerical method that can be applied in both cases, the parabolic case and the hyperbolic case being the later usually more difficult to handle. We also study carefully the discretisation errors.

\section{THE NUMERICAL SCHEME}

In order to remove the $t$-dependent terms, we apply the Laplace transform to the equation (8), where the Laplace transform $\widetilde{C}$ of the mass concentration $C$ is defined by

$$
\widetilde{C}(x, s)=\int_{0}^{\infty} \exp (-s t) C(x, t) d t
$$

We obtain the ordinary differential equation

$$
\frac{d^{2} \widetilde{C}}{d x^{2}}-\lambda_{\theta}^{2} \widetilde{C}+\frac{d}{d x}\left(\frac{P}{D} \widetilde{C}\right)=0
$$

where $\lambda_{\theta}=\left(\left(\theta s^{2}+s\right) / D\right)^{1 / 2}$ and $s$ is a complex variable.

If $P$ is a constant, then we easily have the solution of equation (14):

$$
\widetilde{C}(x, s)=\exp (-(P / 2 D) x)\left(k_{1} e^{b_{\theta} x}+k_{2} e^{-b_{\theta} x}\right),
$$


for $b_{\theta}=\sqrt{(P / D)^{2}+4 \lambda_{\theta}^{2}} / 2$. From the boundary conditions (11) we get

$$
\widetilde{C}(0, s)=\widetilde{g}(s) \text { and } \widetilde{C}(\infty, s)=0
$$

The substitution of these conditions in (15) determine the analytical solution of $C(x, t)$ as follows

$$
\widetilde{C}(x, s)=\widetilde{g}(s) \exp \left(-\left[(P / 2 D)+b_{\theta}\right] x\right)
$$

The next step is to determine $C(x, t)$ by using the numerical inversion of the Laplace transform. We may easily see that, for $t \geq 0$ and each value of $x$,

$$
C(t)=\frac{1}{\pi} \exp (\gamma t) \int_{0}^{\infty} \operatorname{Re}\{\widetilde{C}(s) \exp (i \omega t)\} d \omega
$$

where $s=\gamma+i \omega$. Using the Trapezoidal rule with the step size $h=\pi / T$, for $0<t<2 T$, we obtain the following discretised approximation:

$$
C(t)=\frac{1}{T} \exp (\gamma t)\left[\frac{\widetilde{C}(\gamma)}{2}+\sum_{k=1}^{\infty} \operatorname{Re}\left\{\widetilde{C}\left(\gamma+\frac{i k \pi}{T}\right) \exp \left(\frac{i k \pi t}{T}\right)\right\}\right]-E_{T}
$$

where $E_{T}$ is the discretisation error. Assuming $E_{T}$ sufficiently small, then

$$
C(t) \approx \frac{1}{T} \exp (\gamma t)\left[\frac{\widetilde{C}(\gamma)}{2}+\sum_{k=1}^{\infty} \operatorname{Re}\left\{\widetilde{C}\left(\gamma+\frac{i k \pi}{T}\right) \exp \left(\frac{i k \pi t}{T}\right)\right\}\right]
$$

We now apply the quotient-difference algorithm, proposed in [7], to calculate the series in (20) by the rational approximation in the form of a continued fraction. With the purpose of applying this scheme to (20), set

$$
\sum_{k=0}^{\infty} a_{k} z^{k}=\frac{\widetilde{C}(\gamma)}{2}+\sum_{k=0}^{\infty} \widetilde{C}\left(\gamma+\frac{i k \pi}{T}\right) \exp \left(\frac{i k \pi t}{T}\right)
$$

where $s=\gamma+\frac{i k \pi}{T}, k=0,1, \ldots, a_{0}=\frac{1}{2} \widetilde{C}(\gamma), a_{k}=\widetilde{C}\left(\gamma+\frac{i k \pi}{T}\right)$ and $z=\exp \left(\frac{i \pi t}{T}\right)$.

Let the $M$-th partial fraction $v(z, M)$ be

$$
v(z, M)=d_{0} /\left(1+d_{1} z /\left(1+d_{2} z /\left(1+\cdots+d_{M} z\right)\right)\right) .
$$

where the coefficients $d_{k}$ 's are obtained by recurrence relations from the $a_{k}$ 's. We have that

$$
\sum_{k=0}^{\infty} a_{k} z^{k}=v(z, \infty)=v(z, M)+E_{F}^{M}
$$

where $E_{F}^{M}$ is the truncation error associated to the truncation of the continued fraction. Then

$$
C(t)=\frac{1}{T} \exp (\gamma t) \operatorname{Re}\left\{v(z, M)+E_{F}^{M}\right\}-E_{T}
$$

and the approximation for $C(t)$ is given by

$$
C(t) \approx \frac{1}{T} \exp (\gamma t) \operatorname{Re}\{v(z, M)\} .
$$




\section{DISCRETIZATION ERROR}

We have two errors controlling our discretisation. The first error is related to the integral approximation where

$$
C(t)=C_{T}(t)-E_{T}
$$

for

$$
C_{T}(t)=\frac{1}{T} \exp (\gamma t)\left[\frac{\widetilde{C}(\gamma)}{2}+\sum_{k=1}^{\infty} \operatorname{Re}\left\{\widetilde{C}\left(\gamma+\frac{i k \pi}{T}\right) \exp \left(\frac{i k \pi t}{T}\right)\right\}\right] .
$$

and the error $E_{T}$, according to Crump [8], is given by

$$
E_{T}=\sum_{n=1}^{\infty} e^{-2 n \gamma T} C(2 n T+t) \text {. }
$$

If we assume that our function is bounded such as $|C(t)| \leq M e^{\alpha t}$, the error can be bounded by

$$
E_{T} \leq M e^{\alpha t} \sum_{n=1}^{\infty} e^{-2 n \gamma T}=\frac{M e^{\alpha t}}{e^{2 T(\alpha-\alpha)}-1}, \quad 0<t<2 T .
$$

It follows that by choosing $\gamma$ sufficiently larger than $\alpha$, we can make $E_{T}$ as small as desired. For practical purposes and in order to choose a convenient $\gamma$ we use the stricter inequality which bounds the error

$$
E_{T} \leq M e^{\alpha t} e^{-2 T(a-\alpha)} \text {. }
$$

The second error, $E_{F}$ comes from the approximation of the continued fraction $v(z)$. This error is controled by imposing a tolerance $T O L$ such as

$$
\left|v\left(z, M_{k}\right)-v\left(z, M_{k-1}\right)\right|<T O L
$$

and then we have

$$
C(t) \approx \frac{1}{T} \exp (\gamma t) \operatorname{Re}\left\{A_{M_{k}} / B_{M_{k}}\right\}
$$

In practise it is very difficult to choose an $M$ according to a theoretical result since the analytical truncation errors we are able to get for continued fractions are strongly dependent on the $d_{k}^{\prime}$ s. Although there is a lack of such general results, for some specific test problems we are able to apply some of the theoretical results presented in [9].

\section{FINAL REMARKS}

Additionally to what we describe here, when $P$ is non-constant a spatial discretization needs to be considered and discussed. We have used finite difference schemes for these cases. To analyse the order of convergence of the numerical schemes we have run some test problems. We have also observed how the solution to this problem is affected by changing the parameters $\theta$ and $P$.

\section{REFERENCES}

1. J. Crank, The Mathematics of Diffusion, Oxford University Press, 1974.

2. R. Kubo, M. Toda and N. Hashitsume, Statistical Physics II, Springer Series in Solid-State Sciences, 31 , Springer, 1978.

3. K.C. Liu and H.T. Chen, Numerical analysis for the hyperbolic heat conduction problem under a pulsed surface disturbance, Applied Mathematics and Computation 159, 887-901 (2004).

4. A.K. Das, A non-Fickian diffusion equation, J. Appl. Phys 70, 1355-1358 (1991).

5. A.K. Das, Some non-Fickian diffusion equations: Theory and Applications, Defect and Diffusion Forum 162-163, 97-118 (1998).

6. O.D.L. Strack, A mathematical model for dispersion with a moving front in groundwater, Water Resour. Res. 28(11), 2973-2980 (1992).

7. J. Ahn, S. Kang, Y. Kwon, A flexible inverse Laplace transform algorithm and its application, Computing 71(2), 115-131 (2003).

8. K. Crump, Numerical inversion of Laplace transforms using a Fourier series approximation, Journal of the Association for Computing Machinery 23(1), 89-96 (1976). 9

9. W. Jones and W. Thron, Continued Fractions: Analytic Theory and Applications, Encyclopedia of Mathematics and its Applications, vol. 11, 1980. 